\title{
British Orthodontic Society's initiative on orthodontic retention, A GDP's perspective
}

\author{
P. V. Mc Crory*1
}

\section{Key points}

Discusses the initiative, questions the universality of its message and outlines a number of potential solutions to issues it raises.
Reviews who is currently responsible for post treatment retention
Discusses potential implications for GDPs, DCPs and general dental services providers, including litigation risks.

The British Orthodontic Society (BOS) recently published a number of news articles aimed at drawing attention to its 'Hold that Smile' campaign which promotes lifelong retainer wear. The BOS's stated intention is to 'generate a viral \#HoldthatSmile campaign in order to build awareness of its message that retainers are for life'. The campaign also seeks to recruit the support of GDPs and DCPs, including nurses, in motivating patients to wear retainers for life. It suggests that orthodontists and GDPs need to work collaboratively and suggests that dentists would need to check patients are taking care of their retainers over the long term. This article discusses a number of aspects of the BOS's initiative on retention including the public campaign, the evidence base, long-term retention care, retrospective support, the impact on consent, considerations for GDPs and record-keeping with suggestions on improving long-term support for patients.

\section{The public campaign}

The core message which the BOS is seeking to put across in the campaign is probably best summed up in a statement by the BOS authority on retention, Simon Littlewood:

'I think there are some misconceptions with retention. In the past, we used to think that once patients reached a certain age, their teeth would eventually be stable. Now we know that's not true, there is potential for teeth to keep moving throughout life - it's almost like a normal ageing phenomenon.'

The public campaign has three main elements, a Twitter campaign, two short YouTube hosted information videos produced by the $\mathrm{BOS}$ and news articles published on the BOS website. ${ }^{1-3}$

The Twitter hashtag chosen for the campaign has in fact been in use since at least January

General Dental Practitioner, Cheadle, Stockport, Cheshire SK8 1LN, United Kingdom

${ }^{*}$ Correspondence to: Paul Mc Crory

Email: pvmcch@gmail.com

Refereed Paper. Accepted 1 February 2018

DOI: $10.1038 /$ sj.bdj.2018.227
2012. Up until the beginning of the campaign it appears to have been associated with photography and personal relationship related tweets. ${ }^{4}$ As of 4 November 2017 the author counted approximately 40 tweets which cited the hashtag in relation to orthodontics (the last tweet was on 9 October 2017). Virtually all were posted by professional dental organisations and orthodontic clinics with apparently no significant public engagement.

The videos comprise a 'Hold that smile' animation and a captioned short film which communicate the benefits of using retainers. These are now available for orthodontic and dental clinics to share with their patients (non BOS members are invited to make a contribution to the British Orthodontic Society Foundation). The author does not have information on the extent to which the videos are available in dental clinics. However, the viewing figures for the two videos suggest that there is little engagement with the initiative on YouTube:

- BOS Retention Campaign - Hold that smile; https://www.youtube.com/ watch?v=P5FxothkHMg, 917 views as 7 November 2017
- Hold That Smile - Why are retainers so important? https://www.youtube. com/watch? $\mathrm{v}=5 \mathrm{wCIFjlStzc}, 753$ views as 7 November 2017.

\section{The evidence base}

Both the BOS and the Department of Health (NHS Choices page on orthodontics) ${ }^{5}$ appear to be sending patients the message that if they adhere to a life-long orthodontic retention prescription provided by their orthodontist, then they will have have permanently straight teeth: 'One of the commonest questions I get is "how long do I wear retainers for?" and my answer is "for as long as you want straight teeth"' (BOS)

'The only way to have permanently straight teeth is to wear a retainer on a part-time basis for life." but out also points out that "Changes in the position of your teeth can continue throughout life and are part of the normal ageing process' (NHS Choices).

$\mathrm{O}^{\prime} B r i e n^{6}$ recently reported on a Norwegian study which looked at the stability of orthodontic treatment outcomes in relation to retention status at eight years post treatment. 
Table 1 Stakeholder statements on orthodontic retention identified by the author

\begin{tabular}{l|l} 
Organisation & Position on who is responsible for orthodontic retention
\end{tabular}

The Department of Health:-

The NHS Choices website states 'Under the NHS, your orthodontist is responsible for your care for 12 months after normal treatment ends. After this period, you'll have to pay privately for continuing care, retreatment, and any replacement or repair of retainers.'

NHS England's Guides for commissioning dental specialties - Orthodontics ${ }^{14}$ states on page 31 that 'Patients are fully informed about the risks and benefits of Orthodontic treatment including the likely need for long-term retention to maintain complete alignment.' and on page 33, 'Patient has all necessary information at end of active treatment on retention regime and who to contact should there be a problem.'

The document goes on to describe what the patient should expect at the end of the retention period;

'At discharge from the orthodontic provider, following the supervised retention period, the patient is given all the necessary information regarding on-going management of retention and what they can expect from their GDP. Relapse is often minimal, but all patients differ to the extent that it occurs.'

The associated standards stipulate that the patient and GDP should be provided with a discharge and retention plan. The commissioning guidance appears to imply that the primary responsibility lies with the patient and that the orthodontist should inform the patient what service/s their 'GDP' can provide. The document does not appear to transfer responsibility to the GDS provider.

NHS England

Further guidance on the responsibility of the referring provider/performer is provided on page 50 of the same document; 'NHS contract holders with no orthodontic element within their contract:

Contract holders with no orthodontic element within their contract should manage patients who request the repair or replacement of orthodontic retainers in the following way:

- Within the supervised retention period (up to 12 months after active treatment): Refer back to the orthodontic contractor where treatment was wprovided

- Beyond the supervised retention period: Refer back to the orthodontic contractor where treatment was provided.

The NHS GDS Regulations (2005) do not permit holders of mandatory services contracts to claim UDA activity for the repair/ replacement of orthodontic retainers.'

Therefore, it would appear that prior to providing discharge advice to the patient, it is incumbent on the orthodontist to ascertain whether the referring provider offers orthodontic retainers on a private basis, prior to providing discharge advice to the patient.

The BOS appears to implicitly suggest that the responsibility for post treatment retention lies with the patient.15 The BOS's patient information leaflet on retainers advises patients that:

- 'Retainer wear is your personal responsibility'

- After the 12 month post treatment monitored retention period 'you will have to pay privately for continuing care or re-treatment as well as for any replacement/repair of retainers. Your orthodontist may ask you to sign a retention consent form'

- 'Contact your dentist/orthodontist as soon as possible if you damage or lose your retainer.'

The BOS's authority on retention has also stated, 'asking patients to wear retainers indefinitely adds to the burden of their care. They have to be responsible for wearing and looking after the retainer, as well as getting it checked, repaired and replaced, which may have financial costs.'

A 2008 BOS statement ${ }^{27}$ with the title 'Liability of practitioners for continuing care after completion of active orthodontic treatment', reports the DH's position as 'In most cases under the new regime a 12 month retention period would be about right for but a very few exceptions. Orthodontists should accept the differing requirements of individual patients, and an artificial time limit for supervised retention would interfere with this.'

The British Orthodontic Society

The BOS went on to comment, 'This ruling means that orthodontists have a continuing responsibility for patients for at least twelve months after completion of active treatment, including repairs to retainers. If a patient wishes to continue retention indefinitely the orthodontist has the discretion to make a judgement about the clinical necessity for this. Where the need to extend retention is judged to no longer be "proper and necessary" it is not a part of the NHS contract.'

This appears to imply that in the case of NHS orthodontic care, orthodontists have continuing responsibility for patients for as long as there is a 'proper and necessary' clinical need and that such care is deemed to fall within the NHS contract. However, no IOTN dental health component grade threshold appears to have been specified in this regard. The statement also appears to suggest that after the initial 12 month retention support period, orthodontists are not contractually liable for any deterioration in the cosmetic outcome achieved by the course of treatment. Given that many patients, parents and guardians are likely to be more concerned about the cosmetic outcome, in the opinion of the author, the NHS might wish to draw greater attention to its emphasis on providing care which addresses clinical need. There may also be a case for the NHS adopting a policy which states that improvements in the IOTN aesthetic component during treatment are coincidental, may well be transient and are not formally within the envelope of care provided. Such an approach would provide greater clarity for patients and could reduce the future litigation risk for the profession.

A search of the BDA's website (on 4 November 2017) for the terms 'orthodontic retainer' and 'orthodontic retention' failed to return a result providing a definitive position over the past 12 months.

The British Dental Association

However, in a recent BOS news article, the Vice Chair of the British Dental Association, appears to imply that the responsibility lies with the patient rather than the provider of the orthodontic care or the patient's general dental practitioner: 'In many cases, orthodontic treatment can be a lengthy process, but once it has concluded it can come as a shock that it may be necessary to wear retainers for life. There has been little co-ordinated information for patients on the reasons for this and what they need to do if they break or need to be repaired, and the new campaign by the BOS is a welcome addition to closing this gap.'

The General Dental Council

A search of the GDC's website (on 4 November 2017) for the term 'orthodontic retainer' failed to return definitive guidance. However, one document16 gave a report on a patient complaint which included a charge that the dentist had not provided a lower retainer. The panel considering the case concluded that 'was no complaint to answer in relation to the case.'

The CQC

A search of the CQC's website (on 4 November 2017) for the terms 'orthodontic retainer' and 'orthodontic retention' did not return any position statements or other comments on providers' responsibilities in respect of orthodontic retention.

- Using the the "All Information" option on the search menu. Search date 4-11-17. 
He expressed the opinion that 'our level of knowledge about retention is surprisingly low' and that this particular study may be considered to be the 'best that we can get' in this regard. He concluded, be it with a degree of caution, that:

- The study 'reinforces that relapse is inevitable, particularly in the lower incisors'

- 'The concept of long-term retention may not be the answer to preventing relapse'.

In the 14 September 2017 BOS news article which reported the launching of its 'Hold that Smile Campaign', the BOS authority on retention was reported to have said that 'the classic research on relapse was done on the west coast of the US which followed up patients who only wore retainers for one or two years. They found that 10 years later, $70 \%$ of those needed re-treatment ${ }^{\prime}$ The BOS article appeared to rely upon this research as the evidence base for it advocating life-long retention. However, the article did not discuss the $30 \%$ not requiring re-treatment despite Littlewood ${ }^{7}$ having done so when referencing research from 1988 and 1990, by Little. ${ }^{8,9}$ Also, it did not discuss other factors which could have influenced the development of the occlusion ${ }^{10}$ in the intervening years years including, differential mandibular growth, physiologic mesial drift and occlusions which apply anteriorly-directed forces on mesially-tipped teeth. ${ }^{11}$

In a recent article, Littlewood ${ }^{12}$ made the following comments on orthodontic retention research:-

- 'Most current retention RCTs study periods are in the region of 6-24 months'

- 'Recalling patients years later to measure relapse is difficult.'

He also questioned whether the right questions were being asked in relation to retention

- How much of an issue is late onset lower anterior crowding?

- What are patients' value judgements with regard to the compliance, repair, replacement and relapse cost benefit analysis?

In advocating life-long retention, the BOS also appears to be implicitly suggesting that there is no evidence on factors which can assist in predicting relapse and there is no expectation of progress in this area. However, in a retrospective longitudinal study of 70 post-retention patients with records extending between four and ten years post treatment (T3), González-Gil de Bernabé et al. ${ }^{13}$ identified a number of variables and occlusal features which are associated with significantly increased relapse risks. They also found that at T3, while only $7.1 \%$ of the study group presented absolute stability, $68.6 \%$ presented relative stability (PAR score changes within the \pm 5 range between the end of active treatment and T3).

Therefore, it appears that both the research base and adult facial development factors do not support the broad assertion that life-long retainer use will maintain tooth positions achieved at the completion of active treatment or that relapse is completely unpredictable. Furthermore, the recent BOS initiative on retention does not appear to fully articulate the reservations and thinking of its own authority on retention, and does not provide advice on communicating the revised guidance to past patients who might benefit from it?

It is also worth considering that given current life expectancies, a definitive evidence base on orthodontic retention could take in the region of 100 years to develop.

\section{Who is responsible?}

The BOS initiative has thrown into sharp relief the need for clarity on who is responsible for long-term orthodontic retention. A search for position statements resulted in the findings shown in Table 1.

The question of responsibility is further complicated by the high probability that a patient will have general dental care provided by a series of GDPs over their lifetime.

Under the current model of care provision in England, specialist orthodontic care and general dental services are delivered by providers. The CQC's glossary of terms ${ }^{17}$ states that 'The entire group of people employed for the purposes of carrying on a regulated activity' are defined as an organisation's 'staff'. It could be argued that this biases responsibility for orthodontic retainer care towards the provider. The provider-performer model adds a layer of complexity to the responsibility issue in that, where any long-term liability is deemed to reside with those providing general dental care, then presumably the provider would have responsibility and the performer only where it is a contractual requirement? In the latter circumstances, it would be reasonable to assume that the performer would have access to collated, comprehensive records of the referring dentist, the orthodontist, all the previous providers of care post treatment and all those records generated by any performers who had provided care for the patient while under the care of his/her current provider.

\section{Impact of the new BOS guidance}

Currently the Faculty of General Dental Practice ${ }^{18}$ advises that 'records should ideally be retained for up to 30 years, and for a minimum of 11 years after the completion of treatment.'

The BOS cites the Consumer Protection Act ${ }^{19}$ as the basis for its recommendations on retaining orthodontic radiographs suggesting they be retained until 11 years after the last record entry or 25 years of age, whichever is the longer. However, it acknowledges that some defence and protection organisations advise that from a dento-legal perspective all records should be retained indefinitely but considers this may prove difficult.

As the BOS initiative is in effect suggesting that life-long retention will preserve the aesthetic and functional outcomes achieved at the time of treatment, then it appears to place an obligation on the providers of orthodontic services to retain treatment records for the lifespan of the patient. It would also appear to be the case that this obligation would extend to those providing orthodontic treatment planning and device manufacturing services such as clear aligner producers.

\section{Factors to consider}

In England, those providers of general dental services which choose to offer private orthodontic post treatment retention services may need to consider the following:

- Providers, performers and DCPs participating in the provision of such services would need to ensure that they had indemnity which covers this area of practice

- The need to adopt the Peer Assessment Rating index ${ }^{20}$ or other orthodontic outcome assessment tool in order to audit the quality of service they are providing

- Securing and retaining copies of full orthodontic treatment records, including:

- Cast models or digital impressions of the occlusion at the time of completion of treatment by the orthodontic specialist/ special interest dentist

- Any available information on the assessed relapse risk

- The risk of late onset crowding

- The potential effect of retention and relapse on bruxing behaviour and TMD risk 
o Who provided informed consent in respect of the post treatment retention regime and what are the patient's current wishes and future expectations ${ }^{21}$ given the available personal clinical records

- All orthodontic monitoring records held by other post-treatment providers of retention-related care

- Retain the records for the life-time of the patient and be prepared to provide copies to any future providers who require copies

- Ensure that performers have the necessary training in monitoring occlusal development and restoring occlusions where relapse has occurred

- The cost implications for patients who request this service as an element of their care and/or inclusion of the service in dental insurance schemes

- Contracts of employment/engagement for dentists and DCPs which have opt-in clauses for the provision of retentionrelated care and which fully address the above considerations in order to reduce the risk of long-term liability given recent case law. $^{22}$

Littlewood has suggested that it would be helpful if general dentists could take responsibility for a number of aspects of long-term retention 'if the appropriate training and remuneration system were in place.' These include:

- 'Detecting failed bonded retainers and repairing and replacing them as required

- Monitor the fit of removable retainers and replacing them as required

- Provide motivation to patients to wear and look after their retainers appropriately

- Monitor the effect of retainers on the patient's oral health and intercepting any problems. $^{\text {? }}$

However, the article makes no mention of involving other team members in contrast to the BOS initiative goals. Also, these opinions were published in July 2017, yet they are not referred to in any of the three BOS news articles referenced above.

\section{Future developments in patient care}

The twin approaches of further research into retention and improved patient care may be expected to improve patient outcomes in the medium- to long-term. In the UK initiatives could potentially include the following:
- Direct recording and life-long storage of 3D digital records ${ }^{23}$ of orthodontic outcomes on completion of treatment or indirect 3D scanning from cast models

- Copying of orthodontic records into NHS unified electronic patient records ${ }^{24}$ or other long-term health record storage systems in order to support long-term/ life-long retention where this is indicated and requested by the patient

- Improvements in the methods of assessing patients' understanding of the demands which long-term/life-long retention will place on them including realistic estimates of the full associated financial costs (cost of clinical time, retainers and costs in terms of time to the patient). It is worth bearing in mind that for patients/parents/ guardians from deprived backgrounds, these projected costs could have a disproportionately great deterrence effect on proceeding with treatment

- Long-term clinical research into relapse risk factors using NHS hosted digital occlusal records of participating patients

- High levels of communication between treating orthodontists and general dental service providers who provide post orthodontic discharge orthodontic retainer services.

It has been suggested that smartphones, apps and selfies might provide a route to monitoring long-term post-treatment changes. While such technology may be expected to improve and be capable of creating 3D models of the dental arches and their occlusal relationship, it is unlikely that the analysis will achieve the resolution which clinical devices will achieve. Such an approach would also place significant demands on the patient in terms of accurately recording and long-term monitoring of their occlusal status.

Clear aligner based orthodontic treatments can be used to treat a range of orthodontic cases ${ }^{25}$ and are now commonly provided in general dental practice by non-specialists. Many of the areas of discussion in this paper also apply to those providers and their performers who offer such care. Ironically, the computer supported orthodontic planning and device manufacturing services which underpin many of these treatments may enable greater continuity and quality of support during the retention period as the occlusion records will be stored centrally and in digital formats.

\section{Concluding remarks}

The headline message in the BOS initiative does not appear to reflect the complexities of orthodontic retention and as a result, there appears to be a risk that patients may feel excessively optimistic about the potential for maintaining their orthodontic outcome while simultaneously being pessimistic about the prospects for being able to dispense with retainer/s and concerned about the ongoing financial costs. Rising patient expectations and increased uptake of adult cosmetic dental services appear likely to increase the overall level of concern. This in turn could lead to increased patient dissatisfaction, increased initiation of litigation and a degree of uncertainty surrounding orthodontics in the mind of the general public. These concerns are partially offset by the apparently good prospects for improved research-based understanding of orthodontic relapse. However, orthodontists and those providing orthodontic retainer services, may in time come to believe that a campaign with a more nuanced message would have been preferable.

The professional and contractual relationships between the stakeholders in orthodontic retention care provision do not appear to be well described and as a consequence there appear to be potential risks for both the public and GDC registrants (young colleagues in particular). Therefore, in the opinion of the author, the following actions should be taken to mitigate these risks (where they are not already in train).

All stakeholders (including organisations which represent NHS and non-NHS patient populations) should:

1. Undertake a full assessment of the extent to which patients can reasonably be held personally responsible for relapse. The assessment should consider factors including:

- Reasonable estimates of the likely capacity of different groups within society to be able to afford orthodontic retention services

- The capacity of the patient to comply with post treatment advice

- A statistical analysis of the transient non-compliance risks in otherwise good compliance groups (eg, acute medical and personal circumstances which interrupt compliance)

2. Undertake a full assessment of litigation risks and map them to commissioning bodies, providers, performers and DCPs 
according to the envelope of possible contractual agreements

3. In the case of provider-performer relationships, decide which of the parties should carry the primary risk in order that appropriate indemnity cover can be put in place

4. Assess what residual indemnity cover performers and providers not engaging in the provision of orthodontic retention-related services would still require in order to comply with regulatory requirements.

\section{The CQC should give consideration to requiring that:}

1. General dental service providers clearly state whether or not they provide orthodontic retention-related services

2. Those that offer orthodontic retentionrelated services ensure that they can provide a comprehensive service which is audited regularly

3. No registrants/'staff" are coerced into providing orthodontic retention-related services

4. Providers hold adequate indemnity insurance

5. Those registrants/'staff' who voluntarily contract into undertaking orthodontic retention related services:

- Only provide care when they have full access to all the relevant clinical records

- Have the full envelope of care they provide detailed in optional, explicit contract clauses.

\section{The appropriate regulators should:}

1. Investigate the products and post-active treatment support services provided by clear aligner medical device companies with a view to determining the current distribution of risk between them, providers of dental services and GDC registrants, based upon an analysis of the envelope of contractual arrangements currently in place

2. In the event that the review identifies any areas of uncertainty regarding the risk/s to the public, assess whether their existing regulatory powers are sufficient to secure a resolution of those risks, or if they require additional powers in order to manage them.

\section{The NHS should:}

1. Assess how it might best use orthodontic SNODENT data to inform future orthodontic commissioning decisions which have long-term retention implications for patients
2. Assess the feasibility of hosting $3 \mathrm{D}$ digital copies of treatment outcome occlusal records

3. Assess the potential benefits to patients of hosting other treatment records relating to orthodontic retention including discussions with the patient, care planning and patient consent. The orthodontic service provider would retain responsibility for the secure storage of the record in order to avoid the transfer of liability to the NHS

4. Include mandatory, low cost, fully patient funded, life-long, retainer repair and replacement service items in orthodontic contracts.

\section{The BOS should:}

1. Clarify its position on what retrospective advice should be communicated to patients whose treatment was completed before the start of the 'Hold that Smile' retention campaign. This advice should be communicated to its members and also be published in order for regulators and nonmember providers of orthodontic services to understand the approach it has adopted

2. Assess how its members can improve communications with non-specialist providers of orthodontic retainer services and provide the membership with appropriate guidance

3. Audit and review the quality of orthodontic retention-related patient information services and the degree of success in communicating these successfully to patients. The results should be published in order for other stakeholders to review and comment

4. Consider compiling an actively managed register of orthodontic retention care service providers and making the information available online to assist the public in general, and act as a signposting resource which its members can direct patients to

5. Commission the development of an evidence-based algorithm for estimating the potential long-term, total cost of orthodontic retention services for individual patients. The algorithm would require regular review and appropriate revision to ensure that it continues to reflect the prevailing knowledge base on retention.

\section{Providers of orthodontic services should:}

1. Retain orthodontic care records for as long as it is assessed that the patient may require fixed and/or removable retainers in order to maintain the original orthodontic outcome

2. Prior to beginning a course of orthodontic treatment, provide patients with:
- A written, best guess estimate of the potential full retention period/life-time cost of orthodontic retention services, preferably calculated using an evidencebased algorithm which factors in all known relevant risks

- A written assessment of the likelihood of relapse occurring based upon an analysis of the individual patient's risk

- Written details of any other treatment options which might reasonably be expected to reduce the long-term compliance and financial burdens on the patient

- A written assessment of the likely aesthetic outcome in the event of post-treatment relapse

- Provide patients with and/or direct them to information on the availability of specialist and non-specialist providers of post treatment orthodontic retention care services.

The author hopes that this article will contribute to the debate on the risks surrounding orthodontic retention, how they can be better managed going forward and that, where possible, long-term patient care is underpinned by actively managed, voluntary agreements between stakeholders in order to minimise the need for regulation.

1. British Orthodontic Society. BOS launches 'Hold that Smile' retention campaign. 2017. Available at https:// www.bos.org.uk/News-and-Events/News (accessed November 2017).

2. British Orthodontic Society. 'Hold that smile' film and animation now available for dental clinics to share on their website. 2017. Available at https://www.bos.org uk/News-and-Events/News (accessed November 2017).

3. British Orthodontic Society. British Orthodontic Society launches patient campaign to promote lifelong retainer wear. 2017. Available at https://www.bos.org.uk/Newsand-Events/News (accessed November 2017).

4. Twitter: \#HoldthatSmile. Available at https: //twitter. com/hashtag/holdthatsmile?src=hash (accessed Novem ber 2017).

5. NHS England. NHS Choices. Available at https://www. nhs.uk/conditions/orthodontics/treatments/ (accessed October 2017).

6. O'Brien K. Fixed retainers do not prevent relapse? A long term study. Kevin O'Brien's Orthodontic Blog. 2017. Available at http://kevinobrienorthoblog.com/fixedretainers-long-term-study/ (accessed October 2017).

7. Littlewood S J. Responsibilities and retention. APOS Trends Orthod 2017; 7: 211-214. Available at http://www.apospublications.com/text. asp?2017/7/5/211/214570 (accessed November 2017).

8. Little R M, Riedel R A, Artun J. An evaluation of changes in mandibular anterior alignment from 10 to 20 years postretention. Am J Orthod Dentofacial Orthop 1988; 93: 423-428.

9. Little R M. Stability and relapse of dental arch alignment. Br J Orthod 1990; 17: 235-241.

10. Reyes C A. Extraction of Mandibular Third Molars Does Not Minimize Lower Arch Crowding. 2014. Available at https://cats.uthscsa.edu/found_cats_view. php?id=2652\&vSearch $=$ (accessed November 2017). 
11. Steinnes J, Johnsen G, Kerosuo H. Stability of orthodontic treatment outcome in relation to retention status [abstract]: An 8-year follow-up. Am J Orthod Dentofacial Orthop 2017; 151: 1027-1033.

12. Littlewood S. Orthodontic Retention - finding the answer? Kevin O'Brien's Orthodontic Blog. 2017. Available at http://kevinobrienorthoblog.com/orthodontic-retention/ (accessed October 2017).

13. de Bernabé P G, Montiel-Company J M, ParedesGallardo V, Gandía-Franco J L, Bellot-Arcís C. Orthodontic treatment stability predictors: A retrospective longitudinal study. Angle Orthodont 2017; 87:

$$
\text { 223-229. }
$$

14. NHS England, Chief Dental Officer team. Guide for Commissioning Orthodontics. 2015. Available at https://www.england.nhs.uk/commissioning/ wp-content/uploads/sites/12/2015/09/guid-commsorthodontics.pdf (accessed October 2017).

15. British Orthodontic Society. Patient information leaflet, retainers. 2012. Available at https://www.bos. org.uk/Portals/0/Public/docs/PILs/retainersapril2013. pdf (accessed October 2017).

16. General Dental Council. Annual Review Content Case Study 1. Available at https://www.gdc-uk.org/
api/files/2015-02-03_5(a)\%20Appendix\%201\%20 Draft\%20Annual\%20Review\%20content\%202014. pdf [Accessed 4th November 2017].

17. Care Quality Commission. Guidance for providers on meeting the regulations Annex C: Glossary of terms. 2014. Available at https://www.cqc.org.uk/sites/ default/files/20150210_guidance_for_providers_glossary_of_terms_annex_c.pdf (accessed November 2017).

18. Faculty of General Dental Practice (UK). Clinical Examination \& Record Keeping Standards, Retention of Records. Available at https://www.fgdp.org.uk/openstandards/25-retention-records (accessed October 2017).

19. British Orthodontic Society. Guidelines for the use of Radiographs in Clinical Orthodontics. 2015. Available at https://www.bos.org.uk/portals/0/public/docs/ general $\% 20$ guidance/orthodontic $\% 20$ radiographs $\% 20$ 2016\%20-\%202.pdf (accessed October 2017).

20. British Orthodontic Society. The Peer Assessment Rating (PAR) index. Available at https://www.bos.org. uk/Professionals-Members/Research-Audit/Quality\%20 Assurance\%20in-Orthodontics/The-Peer-AssessmentRating-PAR-index (accessed October 2017).
21. Bright E, D'Cruz L, Milne E. Consent- an update. Br Dent J 2017; 222: 655-657.

22. Jacobs J. Dentistry. Are dental associates employed or self-employed? 2014. Available at http://www.dentistry. co.uk/2014/10/20/associate-classed-employed-selfemployed/ (accessed November 2017)

23. Zhang F, Suh K J, Lee K M. Validity of Intraoral Scans Compared with Plaster Models: An In-Vivo Comparison of Dental Measurements and 3D Surface Analysis. PLOS One 2016; 11: e0157713.

24. Houses of Parliament Parliamentary Office of Science \& Technology. Electronic Health Records. Postnote Number 519 February 2016. Available at http://researchbriefings. files. parliament.uk/documents/POST-PN-0519/POSTPN-0519.pdf (accessed November 2017).

25. British Orthodontic Society. Clear aligners. Available at https://www.bos.org.uk/Public-Patients/Orthodonticsfor-Children-Teens/Treatment-brace-types/Removableappliances/Clear-aligners (accessed November 2017).

26. NHS England. Business Services Authority. Dental Hand book, A Guide for commissioners, practices and dentists in England Available at https://www.nhsbsa.nhs.uk/ sites/default/files/2017-05/Dental\%20handbook\%20 v1.0.pdf Appendix 4, pp87 (accessed March 2018). 